\title{
Estimation of Heterosis for Grain Yield and Yield Components in Pearl Millet (Pennisetum glaucum (L.) R. Br.)
}

\author{
Khushbu Chittora ${ }^{1} *$ and J.A. Patel ${ }^{2}$ \\ ${ }^{1}$ Department of Genetics and Plant Breeding, Anand Agricultural University, \\ Anand -388110, Gujarat, India \\ ${ }^{2}$ Regional Research Station Anand Agricultural University, Anand -388110, Gujarat, India \\ *Corresponding author
}

\section{A B S T R A C T}

\section{Keywords}

Heterobeltiosis, Standard heterosis, Pennisetum glaucum male sterile line.

Article Info

Accepted: 10 February 2017 Available Online: 10 March 2017
An experiment comprised of six male sterile lines, eight inbred testers of pearl millet, their 48 hybrids and two standard check (GHB-538 and GHB-558) was conducted at Regional Research Station, Anand Agricultural University, Anand, Gujarat in Kharif 2014 for studying the extent of hybrid vigour in F1 for grain yield and its components. The cross JMSA-9904 x AIB-15 showed highest and significant standard heterosis for total effective tillers, ear head girth, grain yield per plant and panicle harvest index. Among 48 hybrids studied, three hybrids namely JMSA-9904 x AIB-15, JMSA-9904 x AIB-30 and ICMA99555 x AIB-30 selected as best crosses since they expressed high standard heterosis over standard hybrid for many of the traits studied for high grain yield.

\section{Introduction}

Pearl millet (Pennisetum glaucam (L.) R. Br., $2 \mathrm{n}=2 \mathrm{x}=14$, Family: Poaceae) is warm season, annual, $\mathrm{C}_{4}$ a highly cross-pollinated crop with the advantages of huge genetic variability and availability of efficient cytoplasmic genetic male sterility system. The protogynous flower morphology of pearl millet makes it a highly cross-pollinated crop with extent of out crossing above 85 per cent, and thereby it is highly heterozygous and heterogeneous. In India, it is mainly cultivated in Rajasthan, Uttar Pradesh, Gujarat, Haryana and Maharashtra, which shares about 91.87 per cent of total pearl millet production. Pearl millet occupies an area of 7.20 million hectares with a production of 8.74 million tones and productivity of $1214 \mathrm{~kg} \mathrm{ha}^{-1}$ in the country. While, in Gujarat, it is grown in 1.07 million hectares with a production of 1.23 million tones and productivity of $1226 \mathrm{~kg} \mathrm{ha}^{-1}$ (Anonymous, 2010).

Exploitation of hybrid vigour is considered to be one of the outstanding achievements in this crop. In heterosis breeding program, it is essential to study and evaluate available promising diverse parental lines for their hybrids nicking ability for grain yield and its components. Recognition of a potential hybrid combination through the magnitude 
and direction of heterotic effects is of paramount importance.

\section{Materials and Methods}

Present study involving six CGMS inbred lines viz JMSA 9904, JMSA-101, ICMA08111, ICMA-92777, ICMA-96333 and ICMA-99555,obtained from Main Pearl Millet Research Station, Jamnagar, eight restorer pollinators viz. AIB-6, AIB-10, AIB14, AIB-15, AIB-17, AIB-20, AIB-28 and AIB-30; developed at Regional Research Station, AAU, Anand and two standard check hybrids GHB-538 and GHB-558. The parental lines and testers were crossed in line $\mathrm{x}$ tester mating design during summer 2014, and evaluated in randomized complete block design at the Regional Research Station, Anand Agricultural University, Anand during kharif 2014.

Five competitive plants from each experimental unit of every replication were selected randomly for recording observations on component characters viz., Days to $50 \%$ flowering, days to physiological maturity, plant height $(\mathrm{cm})$,number of total tiller per plant, number of effective tillers per plant, ear head length $(\mathrm{cm})$, ear head girth $(\mathrm{cm})$, ear head weight $(\mathrm{g})$, grain yield per plant, test weight $(\mathrm{g})$,panicle harvest index and protein content $(\%)$. Protein estimation was done with Near Infrared Spectrophotometer. Panicle harvest index was calculated as:

$$
\mathrm{PHI}=\frac{\text { Grains y ield per plant }}{\text { Earheads weight per plant }} \times 100
$$

Analysis of variance was performed to test the significance of difference among the genotypes for the characters studied, as suggested by Panse and Sukhatme, (1957). The expression of heterosis in 48 hybrids involving six CGMS lines and eight testers was measured in terms of heterobeltiosis in relation to better parent and standard heterosis in comparison with standard check GHB-538 and GHB-558.

\section{Results and Discussion}

\section{Analysis of variance}

The analysis of variance for yield and its components traits in RCBD revealed that the mean square values due to genotypes were highly significant for all the characters, which indicated existence of sufficient genetic variability in the experimental material for all the characters (Table 1).

Mean values of grain yield and yield component characters of parents (lines and testers) and their hybrids is presented in table 2. The range of heterobelteiosis and standard heterosis as well as number of hybrids showing significant heterosis in desirable direction is presented in table 3 .

\section{Estimation of $\mathrm{HB}$ and $\mathrm{SH}$}

Heterobeltiosis (HB) and standard heterosis (SH) in negative direction for days to $50 \%$ flowering are desirable for earliness. The hybrid JMSA-101 x AIB-20 (-13.16\%) exhibited the least estimate of $\mathrm{HB}$ followed by hybrids JMSA-9904 x AIB-17 (-12.90\%) and ICMA-99555 x AIB-6 (-11.95\%). None of the hybrids registered significant and negative SH. The results are in accordance with findings of (Arulselvi et al., 2006) and (Patel et al., 2016) for Heterobelteiosis and (Dangaria et al., 2009) and (Snedecor et al., 1967) for SH. Negative estimates of heterobeltiosis (HB) and standard heterosis (SH) for days to physiological maturity of grain are desired. The estimates of $\mathrm{HB}$ ranged from -12.50 (ICMA-99555 x AIB-6) to 7.14 per cent (ICMA-08111 x AIB-28). The cross ICMA-99555 x AIB-6 (-12.50\%) exhibited the least heterotic effect. only two crosses exhibited significant and negative heterotic effect. The results are in conformity with 
reports of $\mathrm{HB}$ as well as $\mathrm{SH}$. None of the hybrid registered significant and negative heterobelteiosis as well as standard heterois for plant height. The minimum and maximum values for heterobeltiosis were -14.29 (JMSA$101 \mathrm{x}$ AIB-17) and 35.59 (JMSA-9904 x AIB-15) per cent for number of total tiller per plant. Only one hybrid, JMSA-9904 x AIB$20(16.67 \%)$ had significant estimates of SH. For number of effective tillers per plant minimum and maximum values for heterobeltiosis were -27.91 (ICMA-08111 x AIB-28) and 44.13 (JMSA-9904 x AIB-17) per cent, respectively. Only one hybrid ICMA-08111 x AIB-28 (13.94\%) was significantly superior over check hybrid GHB-558. The findings are in accordance with reports of (Dangaria et al., 2009) for $\mathrm{HB}$, and with (Patel et al., 2016) and (Vetriventhan et al., 2008) for SH. For ear head length minimum and maximum values of heterobeltiosis were -27.35 (JMSA-101 x AIB-29) to 38.32 (ICMA-08111 x AIB-17) per cent, respectively. Total $37 \mathrm{~F}_{1} \mathrm{~S}$ had significant estimate of $\mathrm{HB}$ of which $27 \mathrm{~F}_{1} \mathrm{~S}$ registered positive $\mathrm{HB}$. The other $\mathrm{F}_{1} \mathrm{~S}$ with high HB were ICMA-92777 x AIB-14 (33.82\%), ICMA-96333 x AIB-14 (32.93\%) and ICMA-08111 x AIB-6 (32.71\%).

The estimates of standard heterosis over check hybrid GHB-538 varied from 11.49 (JMSA-9904 x AIB-20) to 64.28 (JMSA-101 $\mathrm{x}$ AIB-28) per cent. Total $22 \mathrm{~F}_{1} \mathrm{~S}$ exhibited significant heterobeltiosis for ear head girth of which $12 \mathrm{~F}_{1} \mathrm{~S}$ had positive heterotic effect. None of the $\mathrm{F}_{1} \mathrm{~S}$ exhibited significant positive standard heterosis; whereas $20 \mathrm{~F}_{1} \mathrm{~S}$ depicted significant and negative standard heterosis over check hybrid GHB-558 for ear head girth. The results are in agreement with the findings of (Patel et al., 2008) for HB. For ear heads weight per plant the heterobeltiosis varied from -18.27 (JMSA-101 x AIB-10) to 52.37 (ICMA-08111 x AIB-17) per cent. The estimates of heterosis over standard check hybrid GHB-558, varied from -61.00 (ICMA$08111 \times$ AIB-28) to 19.27 (JMSA $101 \times$ AIB6) per cent. Out of 13 significant cross, 5 crosses depicted positive estimates of SH. The results are in agreement with the findings of (Vagadiya et al., 2010). For grain yield per plant heterobeltiosis, the estimates varied from -22.66 (ICMA-08111 x AIB-10) to 93.78 per cent (JMSA-9904 x AIB-30). Total $30 \mathrm{~F}_{1} \mathrm{~S}$ depicted significant heterotic effect, of which, 29 had positive estimates. The $\mathrm{F}_{1}$ JMSA-9904 x AIB-30 (93.78\%) exerted the highest heterobeltiosis followed by ICMA$99555 \times$ AIB-30 (88.16\%), and JMSA-101 x AIB-28 (75.65\%). The estimates of standard heterosis over hybrid GHB-558 varied from 60.30 (ICMA-08111 x AIB-28) to 14.11 (JMSA-9904 x AIB-15) per cent. Out of 22 significant $\quad \mathrm{F}_{1} \mathrm{~S}$, only $3 \quad \mathrm{~F}_{1} \mathrm{~S}$ exhibited significant and positive standard heterosis. The other $\mathrm{F}_{1} \mathrm{~S}$ with significant and positive SH were JMSA-9904 x AIB-30 (13.75\%) and ICMA-99555 x AIB-30 (11.17\%). The results are in accordance with findings of (Arulselvi et al., 2006; Patel et al., 2008, 2016) for HB, as those found positive magnitude of $\mathrm{HB}$, and with reports of Vetriventhan et al., (2008), for $\mathrm{SH}$ as they found negative magnitude of SH.

For dry fodder yield per plant, values of heterobeltiosis varied from -47.74 (ICMA$99555 \times$ AIB-20) to 115.40 (JMSA-9904 xAIB-14) per cent, whereas, estimates of standard heterosis varied from -140.34 (ICMA-99555 x AIB-14) to.22.68 (ICMA96333 x AIB-20) percent. Total 33 hybrids exhibited significant standard heterosis, of which only 10 hybrids exerted positive heterotic effect (Table 4).

The hybrids with high positive standard heterosis were ICMA-96333 x AIB-20(22.68 $\%)$, JMSA-9904 x AIB-15 (19.73\%) and ICMA-96333 x AIB-15 (19.32\%). Crosse ICMA-92777 $x$ AIB-30(104.95 \%) had highest estimate of $\mathrm{HB}$ for test weight. The 
estimates of standard heterosis over better check hybrid GHB-558 varied from -56.86 (ICMA-96333 x AIB-30) to 20.40 per cent (ICMA-08111 x AIB-17). For panicle harvest index the values of heterobelteiosis varied from -14.64 (ICMA-08111 $\mathrm{x}$ AIB-10) to 47.75 (ICMA-92777 x AIB-30) per cent.

Table.1 Analysis of variance for various characters

\begin{tabular}{|l|l|l|l|l|l|l|l|l|}
\hline $\begin{array}{l}\text { Source } \\
\text { of variation }\end{array}$ & $\mathbf{D . F}$ & $\begin{array}{l}\text { Ear } \\
\text { head } \\
\text { Girth }\end{array}$ & $\begin{array}{l}\text { Ear heads } \\
\text { weight per } \\
\text { plant }\end{array}$ & $\begin{array}{l}\text { Grain } \\
\text { yield per } \\
\text { plant }\end{array}$ & $\begin{array}{l}\text { Dry fodder } \\
\text { yield } \\
\text { per plant }\end{array}$ & $\begin{array}{l}\text { Test } \\
\text { weight }\end{array}$ & $\begin{array}{l}\text { Panicle } \\
\text { harvest } \\
\text { index }\end{array}$ & $\begin{array}{l}\text { Total } \\
\text { protein } \\
\text { content }\end{array}$ \\
\hline Replications & 2 & 0.92 & 89.87 & 40.65 & 50.95 & 0.18 & 12.37 & 0.56 \\
\hline Genotypes & 63 & $2.52^{* *}$ & $808.47^{* *}$ & $493.02^{* *}$ & $5976.21^{* *}$ & $10.65^{* *}$ & $190.08^{* *}$ & $2.73^{* *}$ \\
\hline Parents & 13 & $3.83^{* *}$ & $1136.36^{* *}$ & $554.79^{* *}$ & $5491.63^{* *}$ & $4.54^{* *}$ & $269.06^{* *}$ & $5.27^{* *}$ \\
\hline Lines & 5 & $2.67^{* *}$ & $201.63^{* *}$ & $49.85^{* *}$ & $291.23^{*}$ & $1.08^{* *}$ & $222.36^{* *}$ & $5.31^{* *}$ \\
\hline Testers & 7 & $3.81^{* *}$ & $257.48^{* *}$ & $100.46^{* *}$ & $4880.1^{* *}$ & $3.94^{* *}$ & $127.59^{* *}$ & $4.06^{* *}$ \\
\hline $\begin{array}{l}\text { Linesvs.Test } \\
\text { ers }\end{array}$ & 1 & $9.51^{* *}$ & $11962.10^{* *}$ & $\begin{array}{l}62597.97^{*} \\
*\end{array}$ & $35754.36^{* *}$ & $26.10^{* *}$ & $1491.3^{* *}$ & $9.81^{* *}$ \\
\hline Hybrids & 47 & $1.91^{* *}$ & $327.36^{* *}$ & $203.56^{* *}$ & $4081.38^{* *}$ & $5.76^{* *}$ & $108.08^{* *}$ & $2.24^{* *}$ \\
\hline $\begin{array}{l}\text { Parents } \\
\text { Hybrids }\end{array}$ & 1 & $10.46^{* *}$ & $20510.02^{* *}$ & $13854.6^{* *}$ & $111168.5^{* *}$ & $319.53^{* *}$ & $3156.3^{* *}$ & .004 \\
\hline $\begin{array}{l}\text { Checks } \\
\text { Hybrids }\end{array}$ & 1 & $1.31^{* *}$ & 1.11 & 2.66 & 0.001 & $0.870^{* *}$ & 1.57 & 0.001 \\
\hline $\begin{array}{l}\text { Between } \\
\text { Checks }\end{array}$ & 1 & $4.03^{* *}$ & $320.03^{* *}$ & $105.23^{* * *}$ & $1060.54^{* *}$ & $2.53^{* *}$ & 6.36 & 0.001 \\
\hline Error & 126 & 0.30 & 45.62 & 23.41 & 111.73 & 0.122 & 19.88 & 0.19 \\
\hline
\end{tabular}

Table.2 Mean values of lines, testers and hybrids

\begin{tabular}{|l|l|l|l|l|l|}
\hline \multirow{2}{*}{ Traits } & \multicolumn{4}{l}{ Mean values } & \multicolumn{4}{l|}{} \\
\cline { 2 - 6 } & Lines & Testers & Hybrids & CD at 5\% & CV \\
\hline Days to 50 \% flowering & 51.56 & 54.29 & 50.03 & 2.43 & 2.97 \\
\hline Days to maturity & 84.22 & 85.75 & 84.11 & 2.80 & 2.07 \\
\hline Plant height & 125.72 & 178.16 & 196.69 & 15.23 & 5.03 \\
\hline Total number of tillers & 3.93 & 4.33 & 4.82 & 0.76 & 10.16 \\
\hline Total effective tillers per plant & 2.14 & 2.53 & 2.67 & 0.49 & 11.67 \\
\hline Ear head length & 21.44 & 20.77 & 24.75 & 1.78 & 4.65 \\
\hline Ear head girth & 7.59 & 8.56 & 8.71 & 0.91 & 6.25 \\
\hline Ear head weight per plant & 29.70 & 63.80 & 74.30 & 9.82 & 10.94 \\
\hline Grain yield per plant & 14.44 & 39.16 & 49.20 & 7.81 & 10.77 \\
\hline Fodder yield per plant & 67.30 & 159.48 & 159.48 & 17.07 & 7.20 \\
\hline Test weight & 6.51 & 8.10 & 10.56 & 0.55 & 3.45 \\
\hline Panicle harvest index & 49.60 & 61.62 & 66.30 & 7.21 & 6.95 \\
\hline Total protein content & 11.21 & 10.23 & 10.63 & 0.69 & 4.06 \\
\hline
\end{tabular}


Table.3 Range of heterosis and number of crosses showing significant heterosis in desirable direction in pearl millet

\begin{tabular}{|l|l|l|l|l|}
\hline \multirow{2}{*}{ Characters } & Heterosis \% over better parent & Heterosis \% over standard check \\
\cline { 2 - 5 } & Range & $\begin{array}{l}\text { Number } \\
\text { significant } \\
\text { crosses }\end{array}$ & $\begin{array}{l}\text { Rumber } \\
\text { significant } \\
\text { Crosses }\end{array}$ \\
\hline Days to $50 \%$ flowering & -13.16 to 8.28 & 22 & -3.65 to 24.09 & - \\
\hline Days to maturity & -12.50 to 7.14 & 14 & -4.57 to 12.03 & 2 \\
\hline Plant height & 15.36 to 172.42 & - & -7.74 to 30.52 & - \\
\hline Total number of tillers & -14.29 to 35.59 & 12 & 15.28 to 16.67 & 1 \\
\hline Total no of effective tillers & -27.91 to 44.13 & 7 & -27.91 to 13.94 & 1 \\
\hline Ear head length & -27.35 to 38.52 & 27 & -11.49 to 64.28 & 26 \\
\hline Ear head girth & -30.05 to 27.80 & 12 & -28.49 to 4.46 & - \\
\hline Ear head weight per plant & -18.27 to 53.37 & 24 & -61.00 to 19.27 & 5 \\
\hline Grain yield per plant & -22.66 to 93.78 & 29 & -60.30 to 14.11 & 3 \\
\hline Fodder yield per plant & -47.74 to 115.40 & 31 & -140.30 to 22.68 & 33 \\
\hline Test weight & -8.55 to 104.95 & 45 & -56.86 to 20.40 & 15 \\
\hline Panicle harvest index & -14.64 to 47.75 & 15 & -56.41 to 6.92 & - \\
\hline Total protein content & -21.65 to 33.83 & 9 & -14.00 to 19.63 & 9 \\
\hline
\end{tabular}

Table.4 Top five best heterotic crosses for various characters

\begin{tabular}{|c|c|c|c|}
\hline Characters & $\begin{array}{l}\text { Per se performance of } \\
\text { hybrids }\end{array}$ & HB & $\mathrm{SH}$ \\
\hline $\begin{array}{l}\text { Days to } 50 \% \\
\text { flowering }\end{array}$ & $\begin{array}{l}\text { JMSA-101 x AIB-20 } \\
\text { JMSA-9904 x AIB-17 } \\
\text { ICMA-99555 x AIB-6 } \\
\text { JMSA-9904 x AIB-15 } \\
\text { JMSA-101 x AIB-28 }\end{array}$ & $\begin{array}{l}\text { JMSA-101 x AIB-20 } \\
\text { JMSA-9904 x AIB-17 } \\
\text { ICMA-99555 x AIB-6 } \\
\text { JMSA-9904 x AIB-15 } \\
\text { ICMA-99555 x AIB-15 }\end{array}$ & $\begin{array}{l}\text { JMSA-101 x AIB-20 } \\
\text { JMSA-9904 x AIB-17 } \\
\text { ICMA-99555 x AIB-6 } \\
\text { JMSA-9904 x AIB-15 } \\
\text { JMSA-101 x AIB-28 }\end{array}$ \\
\hline $\begin{array}{ll}\begin{array}{l}\text { Days } \\
\text { physiological }\end{array} & \\
\text { maturity } & \text { of } \\
\text { grains } & \end{array}$ & $\begin{array}{l}\text { JMSA-9904 x AIB-15 } \\
\text { JMSA-101 x AIB-20 } \\
\text { ICMA-92777 x AIB-6 } \\
\text { ICMA-96333 x AIB-6 } \\
\text { ICMA-99555 x AIB-17 }\end{array}$ & $\begin{array}{l}\text { ICMA-96555 x AIB-6 } \\
\text { JMSA-9904 x AIB-15 } \\
\text { JMSA-101 x AIB-20 } \\
\text { ICMA-92777 x AIB-6 } \\
\text { ICMA-96333 x AIB-6 }\end{array}$ & $\begin{array}{l}\text { JMSA-101 x AIB-20 } \\
\text { JMSA-9904 x AIB-15 } \\
\text { ICMA-92777 x AIB-6 } \\
\text { ICMA-96333 x AIB-6 } \\
\text { ICMA-99555 x AIB-17 }\end{array}$ \\
\hline Plant height & $\begin{array}{l}\text { JMSA-9904 x AIB-6 } \\
\text { ICMA-96333 x AIB-28 } \\
\text { JMSA-9904 x AIB-20 } \\
\text { JMSA-9904 x AIB-28 } \\
\text { ICMA-96333 x AIB-6 }\end{array}$ & $\begin{array}{l}\text { JMSA-101 x AIB-15 } \\
\text { JMSA-9904 x AIB-20 } \\
\text { JMSA-9904 x AIB-28 } \\
\text { JMSA-101 x AIB-28 } \\
\text { JMSA-101 x AIB-28 }\end{array}$ & $\begin{array}{l}\text { JMSA-9904 x AIB-6 } \\
\text { ICMA-96333 x AIB-28 } \\
\text { JMSA-9904 x AIB-20 } \\
\text { JMSA-9904 x AIB-28 } \\
\text { ICMA-96333 x AIB-6 }\end{array}$ \\
\hline $\begin{array}{l}\text { Number of total } \\
\text { tillers per plant }\end{array}$ & $\begin{array}{l}\text { JMSA-9904 x AIB-20 } \\
\text { ICMA-08111 x AIB-14 } \\
\text { JMSA-9904 x AIB-15 } \\
\text { JMSA-101 x AIB-14 } \\
\text { ICMA-92777 x AIB-6 }\end{array}$ & $\begin{array}{l}\text { JMSA-9904 x AIB-15 } \\
\text { ICMA-92777 x AIB-6 } \\
\text { JMSA-101 x AIB-14 } \\
\text { JMSA-9904 x AIB-6 } \\
\text { ICMA-92777 x AIB-14 }\end{array}$ & $\begin{array}{l}\text { JMSA9904 x AIB-20 } \\
\text { JMSA-9904 x AIB-15 } \\
\text { ICMA-92777 x AIB-6 } \\
\text { JMSA-101 x AIB-14 } \\
\text { ICMA-92777 x AIB-6 }\end{array}$ \\
\hline $\begin{array}{lr}\begin{array}{l}\text { Number } \\
\text { effective }\end{array} & \text { of } \\
\text { per plant } & \end{array}$ & $\begin{array}{l}\text { JMSA-9904 x AIB-15 } \\
\text { JMSA-101 x AIB-20 } \\
\text { ICMA-96333 x AIB-28 } \\
\text { JMSA-101 x AIB-14 } \\
\text { ICMA-92777 x AIB-28 }\end{array}$ & $\begin{array}{l}\text { JMSA-9904 x AIB-17 } \\
\text { ICMA-96333 xAIB-17 } \\
\text { ICMA-08111 x AIB-17 } \\
\text { ICMA-92777 x AIB-17 } \\
\text { JMSA-9904 x AIB-14 }\end{array}$ & $\begin{array}{l}\text { JMSA } 9904 \text { x AIB-15 } \\
\text { JMSA-101 x AIB-20 } \\
\text { ICMA-96333x AIB-28 } \\
\text { JMSA-101 x AIB-14 } \\
\text { ICMA-92777 x AIB-28 }\end{array}$ \\
\hline
\end{tabular}




\begin{tabular}{|c|c|c|c|}
\hline $\begin{array}{l}\text { Average } \\
\text { internode length }\end{array}$ & $\begin{array}{l}\text { ICMA-96333 x AIB-28 } \\
\text { ICMA-96333 x AIB-30 } \\
\text { JMSA-9904 x AIB-20 } \\
\text { ICMA-92777 x AIB-14 } \\
\text { ICMA-99555 x AIB-28 }\end{array}$ & $\begin{array}{l}\text { JMSA-101 x AIB-10 } \\
\text { ICMA-92777 x AIB-10 } \\
\text { ICMA-92777 x AIB-17 } \\
\text { ICMA-92777 x AIB-15 } \\
\text { JMSA-101 x AIB-20 }\end{array}$ & $\begin{array}{l}\text { ICMA-96333 x AIB-28 } \\
\text { ICMA-96333 x AIB-30 } \\
\text { JMSA-9904 x AIB-20 } \\
\text { ICMA-92777 x AIB-14 } \\
\text { ICMA-99555 x AIB-28 }\end{array}$ \\
\hline Earhead length & $\begin{array}{l}\text { JMSA-101 x AIB-28 } \\
\text { JMSA-101 x AIB-20 } \\
\text { JMSA-101x AIB-30 } \\
\text { ICMA-08111 x AIB-17 } \\
\text { JMSA-101 x AIB-6 }\end{array}$ & $\begin{array}{l}\text { ICMA-08111x AIB-17 } \\
\text { ICMA-92777 x AIB-14 } \\
\text { ICMA-96333 x AIB-14 } \\
\text { JMSA-9904 x AIB-15 } \\
\text { ICMA-08111x AIB-6 }\end{array}$ & $\begin{array}{l}\text { JMSA-101 x AIB-28 } \\
\text { JMSA-101 x AIB-20 } \\
\text { JMSA-101x AIB-30 } \\
\text { ICMA-08111x AIB-17 } \\
\text { JMSA-101 x AIB-6 }\end{array}$ \\
\hline Earhead girth & $\begin{array}{l}\text { JMSA-9904 x AIB-15 } \\
\text { ICMA-92777 x AIB-14 } \\
\text { JMSA-9904 x AIB-30 } \\
\text { ICMA-92777 x AIB-30 } \\
\text { ICMA-08111 x AIB-6 }\end{array}$ & $\begin{array}{l}\text { ICMA-08111 x AIB-6 } \\
\text { ICMA-92777 x AIB-14 } \\
\text { ICMA-96333 x AIB-6 } \\
\text { ICMA-92777 x AIB-17 } \\
\text { ICMA-92777 x AIB-30 }\end{array}$ & $\begin{array}{l}\text { JMSA-9904 x AIB-15 } \\
\text { ICMA-92777 x AIB-14 } \\
\text { JMSA-9904 x AIB-30 } \\
\text { ICMA-92777 x AIB-30 } \\
\text { ICMA-08111 x AIB-6 }\end{array}$ \\
\hline $\begin{array}{l}\text { Ear heads } \\
\text { weight per plant }\end{array}$ & $\begin{array}{l}\text { JMSA-101 x AIB-6 } \\
\text { ICMA-99555 x AIB-30 } \\
\text { ICMA-08111 x AIB-17 } \\
\text { JMSA-9904 x AIB-15 } \\
\text { JMSA-101 x AIB-28 }\end{array}$ & $\begin{array}{l}\text { ICMA-08111 x AIB-17 } \\
\text { JMSA-9904 x AIB-15 } \\
\text { JMSA-101 x AIB-28 } \\
\text { JMSA-101 x AIB-6 } \\
\text { ICMA-96333 x AIB-15 }\end{array}$ & $\begin{array}{l}\text { JMSA-101 x AIB-6 } \\
\text { ICMA-99555 x AIB-30 } \\
\text { ICMA-08111 x AIB-17 } \\
\text { JMSA-9904 x AIB-15 } \\
\text { JMSA-101 x AIB-28 }\end{array}$ \\
\hline $\begin{array}{l}\text { Grain yield per } \\
\text { plant }\end{array}$ & $\begin{array}{l}\text { JMSA-9904 x AIB-15 } \\
\text { JMSA-9904x AIB-30 } \\
\text { ICMA-99555 x AIB-30 } \\
\text { JMSA-101 x AIB-28 } \\
\text { ICMA-08111 x AIB-17 }\end{array}$ & $\begin{array}{l}\text { JMSA-9904 x AIB-30 } \\
\text { ICMA-99555 x AIB-30 } \\
\text { JMSA-101 x AIB-28 } \\
\text { JMSA-101 x AIB-30 } \\
\text { ICMA-92777 x AIB-30 }\end{array}$ & $\begin{array}{l}\text { JMSA-9904 x AIB-15 } \\
\text { JMSA-9904x AIB-30 } \\
\text { ICMA-99555 x AIB-30 } \\
\text { JMSA-101 x AIB-28 } \\
\text { ICMA-08111 x AIB-17 }\end{array}$ \\
\hline $\begin{array}{l}\text { Dry fodder yield } \\
\text { per plant }\end{array}$ & $\begin{array}{l}\text { ICMA-96333 x AIB-20 } \\
\text { JMSA-9904 xAIB-15 } \\
\text { ICMA-963333 x AIB-15 } \\
\text { ICMA-08111 x AIB-17 } \\
\text { JMSA-101 x AIB-28 }\end{array}$ & $\begin{array}{l}\text { JMSA-9904 x AIB-14 } \\
\text { JMSA-101 x AIB-14 } \\
\text { ICMA-96333 x AIB-14 } \\
\text { JMSA-9904 x AIB-15 } \\
\text { ICMA-08111 x AIB-17 }\end{array}$ & $\begin{array}{l}\text { ICMA-96333 x AIB-20 } \\
\text { JMSA-9904 xAIB-15 } \\
\text { ICMA-963333 x AIB-15 } \\
\text { ICMA-08111 x AIB-17 } \\
\text { JMSA-101 x AIB-28 }\end{array}$ \\
\hline Test weight & $\begin{array}{l}\text { ICMA-08111 x AIB-17 } \\
\text { ICMA-92777x AIB-17 } \\
\text { ICMA-08111 x AIB-15 } \\
\text { ICMA-92777x AIB-6 } \\
\text { ICMA-92777x AIB-30 }\end{array}$ & $\begin{array}{l}\text { ICMA-92777 x AIB-30 } \\
\text { ICMA-08111 x AIB-30 } \\
\text { ICMA-96333 x AIB-14 } \\
\text { JMSA-9904 x AIB-15 } \\
\text { ICMA-08111 x AIB-14 }\end{array}$ & $\begin{array}{l}\text { ICMA-08111 x AIB-17 } \\
\text { ICMA-92777x AIB-17 } \\
\text { ICMA-08111 x AIB-15 } \\
\text { ICMA-92777x AIB-6 } \\
\text { ICMA-92777x AIB-30 }\end{array}$ \\
\hline $\begin{array}{l}\text { Panicle harvest } \\
\text { index }\end{array}$ & $\begin{array}{l}\text { JMSA-9904 x AIB-15 } \\
\text { JMSA-101 x AIB-14 } \\
\text { JMSA-9904 x AIB-30 } \\
\text { ICMA-99555 x AIB-14 } \\
\text { ICMA-0811 x AIB-15 }\end{array}$ & $\begin{array}{l}\text { ICMA-92777 x AIB-30 } \\
\text { ICMA-08111 x AIB-30 } \\
\text { JMSA-101 x AIB-30 } \\
\text { JMSA-9904 x AIB-30 } \\
\text { ICMA-96333 x AIB-30 }\end{array}$ & $\begin{array}{l}\text { JMSA-9904 x AIB-15 } \\
\text { JMSA-101 x AIB-14 } \\
\text { JMSA-9904 x AIB-30 } \\
\text { ICMA-99555 x AIB-14 } \\
\text { ICMA-0811xAIB-15 }\end{array}$ \\
\hline $\begin{array}{l}\text { Total protein } \\
\text { content }\end{array}$ & $\begin{array}{l}\text { JMSA-101 x AIB-10 } \\
\text { JMSA-101 x AIB-20 } \\
\text { JMSA-101 x AIB-15 } \\
\text { ICMA-92777 x AIB-20 } \\
\text { ICMA-96333 x AIB-20 }\end{array}$ & $\begin{array}{l}\text { ICMA-92777 x AIB-30 } \\
\text { ICMA-92777 x AIB-10 } \\
\text { JMSA-101 x AIB-30 } \\
\text { ICMA-08111 x AIB-6 } \\
\text { ICMA-08111 x AIB-15 }\end{array}$ & $\begin{array}{l}\text { JMSA-101 x AIB-10 } \\
\text { JMSA-101 x AIB-20 } \\
\text { JMSA-101 x AIB-15 } \\
\text { ICMA-92777 x AIB-20 } \\
\text { ICMA-96333 x AIB-20 }\end{array}$ \\
\hline
\end{tabular}

The estimates of SH ranged from -56.41 (JMSA$101 \times$ AIB-15) to 6.92 (JMSA-9904 x AIB-15) per cent. The results are in accordance with the findings of (Dangaria et al., 2009) for HB. The estimates of heterobeltiosis for protein content ranged from -22.23 (ICMA-08111x AIB-28) to 33.83 (ICMA-92777 x AIB-30) per cent. Total 28 $\mathrm{F}_{1} \mathrm{~S}$ had significant estimates; of which, only 9
$\mathrm{F}_{1} \mathrm{~S}$ depicted positive effect. The estimates of standard heterosis over check hybrid GHB-558 varied from -14.00 (JMSA-9904 x AIB-28) to 19.63 (JMSA-101 x AIB-10) per cent. Out of 48 $\mathrm{F}_{1} \mathrm{~S}$, total $18 \mathrm{~F}_{1} \mathrm{~S}$ exhibited significant estimates, of which $9 \mathrm{~F}_{1} \mathrm{~S}$ had negative heterotic effect.

In conclusion, the heterosis calculated over 
better parent and standard check revealed superiority of some cross combinations .For grain yield, the cross JMSA-9904 x AIB-15 $(14.11 \%)$ showed highest significant and positive heterosis over standard check. The other $\mathrm{F}_{1} \mathrm{~S}$ with significant and positive $\mathrm{SH}$ were JMSA-9904 x AIB-30 (13.75\%) and ICMA-99555 x AIB-30 (11.17\%). Crosse, JMSA-9904 x AIB-30 (93.78\%) exerted the highest heterobeltiosis followed by ICMA99555 x AIB-30 (88.16\%), and JMSA-101 x AIB-28 (75.65\%). A perusal of heterosis indicated that hybrids JMSA-9904 x AIB-15, JMSA-9904 x AIB-30 and ICMA-99555 $x$ AIB-30 were found promising for further evaluation. Heterosis is also useful to decide the direction of future breeding programme and to identify the cross combinations which are promising in conventional breeding programme. While interpreting the results, positive effects were considered as favourable effects for all the characters excepts days to flowering, days to maturity and plant height for which negative effects were considered favourable.

\section{References}

Anonymous. 2010. All-India area, production and yield of bajra from 1950-51 to 2009-2010 along with percentage coverage under irrigation. State wise yield of Bajra. Directorate of Economics and Statistics, Department of Agriculture and Cooperation, $1999-2000$ to $2009-10$ as per Final Forecast Reports; 2012.

Arulselvi, S., Mohanasundaram, K., Selvi, B. and Malarvizhi, P. 2006. Heterosis for grain yield components and grain quality characters in pearl millet. ISMN, 47: 36-38
Athwal, D.S. 1965. Hybrid Bajra-1 marks a new era. Indian Farming, 15: 6-7.

Burton, G.W. 1983. Breeding pearl millet. Plant Breed Rev., 1: 162-82.

Dangaria, C.J., Chotalia, J.M., Savaliya, J.J., Davda, B.K. and Pansuriya, A.G. 2009. Hybrid vigour studies in ten newly developed restorer lines of pearl millet (Pennisetumglaucum(L.) R. Br.). Agric. Sci. Digest, 29(4): 275-278.

Panse, V.G. and Sukhatme, P.V. 1978. Statistical Methods for Agricultural Workers. ICAR Publication ( $2^{\text {nd }}$ Ed.), New Delhi.

Patel, K.Y., Kulkarni, G.U. and Patel, D.R. 2008.Heterosis and combining ability studies for grain yield and its components in pearl millet. J. Maharashtra Agric. Univ., 33(1): 012-015.

Patel, B.C., Doshi, J.S. and Patel, J.A. 2016. Heterosis for grain yield components in pearl millet (Pennisetum glaucum (L.) R. Br). Innovare J. Agri. Sci., 4(3): 1-3.

Patil, C.M., Aher, R.P., Anarase, S.S. and Suryawansi, N.V. 2008. Heterosis for grain yield and its components in pearl millet. $J$. Maharashtra Agric. Univ., 33(1): 4-6.

Snedecor, G.W. and Cochran, W.G. 1967. Statistical Methods, 6th. Ed., Pub. By: The Iowa State University Press.

Vagadiya, K.J., Dhedhi, K.K., Joshi, H.J. Bhadelia, A.S. and Vekariya, H.B. 2010. Studies on heterosis in pearl millet.(Pennisetumglaucum (L.) R. Br.). Agric. Sci. Digest, 30(3): 197-201.

Vaghasiya, V.D., Dangariya, C.J. and Dhedhi, K.K. 2009. Heterosis studies in $B \times x$ crosses for selection of superior females for A-line development in pearl millet. Agric. Sci. Digest, 29(2): 84-88.

Vetriventhan, M., Kumari, A.N. and Ganapathy, S. 2008. Heterosis for grain yield components in pearl millet (Pennisetum glaucum (L.) R. Br.). World J. Agric. Sci., 4(5): 657-66.

\section{How to cite this article:}

Khushbu Chittora and Patel, J.A. 2017. Estimation of Heterosis for Grain Yield and Yield Components in Pearl Millet (Pennisetum glaucum (L.) R. Br.). Int.J.Curr.Microbiol.App.Sci. 6(3): 412-418. doi: https://doi.org/10.20546/ijcmas.2017.603.047 